УДК 004.942+519.816+519.711.3

01.00.00 Физико-математические науки

АВТОМАТИЗАЦИЯ РЕШЕНИЯ
СИСТЕМНЫХ ЗАДАЧ МЕТОДОМ
СТРУКТУРИРОВАННЫХ СИСТЕМ
СИСТЕМОЛОГИИ

Синельникова Татьяна Ибрагимовна аспирант

ФГБОУ ВО «Кубанский государственный университет», Краснодар, Россия, fbogin@mail.ru

В статье производится обзор метода структурированных систем системологии, применяемых для решения системных задач. Изложен авторский модифицированный алгоритм структурирования систем Дж. Клира.

Представлен программный модуль, реализующий модифицированный алгоритм структурирования систем

Ключевые слова: СИСТЕМОЛОГИЯ, СИСТЕМНЫЕ ЗАДАЧИ, СТРУКТУРИРОВАННЫЕ СИСТЕМЫ, ПРОГРАММНЫЙ МОДУЛЬ РЕШЕНИЯ СИСТЕМНЫХ ЗАДАЧ

Doi: 10.21515/1990-4665-121-128
UDC 004.942+519.816+519.711.3

Physics and mathematics

\section{AUTOMATION OF SYSTEM PROBLEMS SOLVING BY STRUCTURED SYSTEMS SYSTEMOLOGY}

\author{
Sinelnikova Tatiana Ibragimovna \\ postgraduate student \\ Kuban State University, Krasnodar, Russia, \\ fbogin@mail.ru
}

The article reviews a method of systems structuring systemology for systems problem solving. The author's modified algorithm of systems structuring of G.J. Klir's is presented. It shows software module realizing the modified algorithm of systems structuring

Keywords: SYSTEMOLOGY, SYSTEM PROBLEMS, STRUCTURED SYSTEMS, THE SOFTWARE MODULE OF SYSTEMS PROBLEM SOLVING

В настоящее время системный подход применяется во многих научных областей, при этом данные о работе элементов системы представляют собой полученные путем измерения (наблюдения или определения) состояния этих элементов. Для прогнозирования неустойчивого состояния и оперативного принятия решения относительно мер стабилизации работы системы требуется применение математических методов, основанных на обработке данных о ее функционировании, что зачастую затруднено в виду неформализованности, нелинейности процесса функционирования системы, нестандартности закона распределения.

Возникает необходимость разработки и внедрения математических методов и моделей, общих для различных систем, и создания программных продуктов поддержки принятия решений в различных предметных 
областях. Отправной точкой для развития универсальных методов стали работы [1-7].

Одним из методов решения системных задач является метод структурированных систем Дж. Клира $[8,9]$, который оперируют такими понятиями как подсистема, структурированная система, полная система. Статус системы как полной системы или подсистемы не является абсолютным. Так некоторая система с поведением на одном этапе исследования выступает в качестве элемента структурированной системы, а на другом - как полная система, подсистемы которой образуют структурированную систему. Такая двойственность позволяет полную систему представить в виде иерархии структурированных систем.

При исследовании систем и решении системных задач решаются две основные взаимодополняющие задачи: задача идентификации и задача реконструкции.

Задача идентификации содержит в себе две подзадачи. Первая задача связана с нахождением множества всех полных систем представленных структурированной системой. Вторая задача - это задача выбора из реконструктивного семейства такой полной системы, которая задает лучшую гипотезу относительно реальной полной системы.

Для выбора единственной системы из реконструктивного семейства применяется два подхода:

1. Поиск несмещенной реконструкиии - полной системы, которая опирается на всю информацию, содержащуюся в структурированной системе, и не использует никакой дополнительной информации;

2. Поиск реконструкции с наименьшим риском - полной системы, для которой минимизирована наибольшая возможная ошибка, т.е. обобщенное расстояние между распределениями реконструированной и истинной системы. 
Реконструктивная гипотеза представляет собой набор подсистем, которые удовлетворяют требованию неизбыточности и условию покрытия. Таким образом, реконструктивная гипотеза - это структурированная система с поведением, сравнимая с полной системой с поведением. Несмещенная реконструкиия является реконструктивной гипотезой, выступающей в качестве предполагаемой полной системы. Для определения потери информации при реконструкции полной системы вычисляется шенноновская энтропия. Однако Дж. Клиру удалось разработать вычислительные процедуры, которые заменяют более сложное вычисление энтропии и называются процедурами соединения. Несмещенная реконструкция, если она существует, находится с помощью базовой процедуры соединения. Рассмотрим принцип базовой процедуры.

Пусть дана структурированная система с поведением $\boldsymbol{S} F$ и требуется выполнить операцию соединения для двух ее подсистем, описываемых функциями ${ }^{1} f$ и ${ }^{2} f$ с множеством выборочных переменных ${ }^{1} S$ и ${ }^{2} S$ соответственно. Операция соединения обозначается как ${ }^{1} f *{ }^{2} f$. Все множество выборочных переменных, входящих в состав этих двух подсистем, делится на три подмножества:

$A$ - множество выборочных переменных, входящих только в первую подсистему;

$B$ - множество выборочных переменных, общих для обеих подсистем;

$C$ - множество выборочных переменных, входящих только во вторую подсистему.

Обозначим буквами $a, b, c$ отдельные состояния выборочных переменных, из множеств $A, B, C$ соответственно. Для определения несмещенной реконструкции операция базового соединения должна быть 
выполнена для пар элементов этой системы. При каждом ее выполнении два элемента объединяются в новую структурированную систему. Результат базовой процедуры соединения при вероятностной функции распределения:

общий случай

$$
\begin{aligned}
& A=\emptyset \\
& B=\emptyset
\end{aligned}
$$$$
\left[{ }^{1} f *{ }^{2} f\right](a, b, c)={ }^{1} f(a, b) \cdot{ }^{2} f(c \mid b)
$$

$$
\left[{ }^{1} f *{ }^{2} f\right](b, c)={ }^{1} f(b) \cdot{ }^{2} f(c \mid b)
$$

$$
\left[{ }^{1} f *{ }^{2} f\right](a, c)={ }^{1} f(a) \cdot{ }^{2} f(c)
$$

Если результат применения базовой процедуры соединения $\left[f \downarrow{ }^{x} S\right]={ }^{x} f$ для всех $\left(x \in N_{q}\right)$, то это несмещенная реконструкция, в противном случае $f$ не соответствует заданной структурированной системе и должна быть уточнена итеративной процедурой соединения.

Итеративная процедура соединения. Дана локально согласованная структурированная система с поведением $\boldsymbol{S F}$ с вероятностными функциями поведения ${ }^{j} f\left(j \in N_{0, q-1}\right)$. Также дана функция $f$, полученная с помощью базовой процедуры соединения, примененной к $\boldsymbol{S} F$, и число $\Delta \in[0,1]$. Требуется с точностью до $\Delta$ определить функцию поведения несмещенной реконструкции. Если после выполнения итеративной процедуры соединения сумма $\quad \Sigma_{\mathrm{c}} f_{i}(c)=1, \quad$ то $\left(f^{S F}(c)-\Delta\right) \geq f_{i}(c) \leq\left(f^{S F}(c)+\Delta\right)$, для всех $c \in C$. В противном случае данная структурированная система $\boldsymbol{S} F$ глобально не согласована и реконструкции для нее не существует, а $\boldsymbol{S} \boldsymbol{F}$ бессодержательна. 
Задача реконструкции формулируется следующим образом. Дана система с поведением, рассматриваемая как полная система. Требуется определить, какие наборы ее подсистем подходят для реконструкции заданной системы с заданной точностью. В задаче реконструкции несмещенная реконструкция описывает реконструктивные возможности рассматриваемой гипотезы относительно заданной полной системы. Чем ближе несмещенная реконструкция к заданной системе, тем лучше гипотеза.

Полная система, набор всех ее реконструктивных гипотез с отношением уточнения, образующим решетку, называется решеткой уточнения. Решетка уточнения может быть получена выполнением одной универсальной процедуры уточнения для любой указанной реконструктивной гипотезы. Реконструктивные гипотезы, формируются из полной системы в несколько шагов и подразделяются на уровни уточнения, включающие решетки уточнения.

Любая реконструктивная гипотеза полностью описывается:

- семейством подмножеств входящих в нее переменных;

- функциями поведения, соответствующими отдельным подмножествам переменных.

Если опустить свойство 2), то свойство 1) определяет класс инвариантности реконструктивных гипотез, отличающихся друг от друга только функциями поведения их элементов. Этот класс инвариантности называется структурой.

Для множества переменных $S$ множество структур, представляющих все реконструктивные гипотезы любой полной системы, определенной на $S$, состоит из семейств подмножеств $S$, удовлетворяющих условиям неизбыточности и покрытия. Обозначим все множества переменных одной мощности $n$, общим множеством структур $G_{n}$, определенным на множестве 
$N_{n}$ натуральных чисел. Формально для любого $n \in N$, $G_{n}=\left\{G_{i} \mid G_{i} \subset P\left(N_{n}\right)\right\}, \quad G_{i}-$ удовлетворяет условиям неизбыточности и покрытия.

В этом формальном определении через $G_{i}$ обозначены элементы $G_{n}$, являющиеся наиболее общими структурами, рассматриваемыми при решении задачи реконструкции. Индекс $i$ идентифицирует структуры из $G_{n}$, и обычно $i \in N_{\left|G_{n}\right|}$. Структуры из множеств $G_{n}$ называются $G$ структурами.

Основным вопросом задачи реконструкции является разработка эффективных вычислительных процедур, допускающих представление, оценку и сравнение реконструктивных гипотез, представленных всеми структурами для заданного множества переменных. Для ее успешного решения требуется использовать соответствующее упорядочение и классификацию структур.

Определим упорядочение структур. Пусть даны две структуры $G_{i} \in G_{n}$ и $G_{j} \in G_{n}$. Будем называть $G_{i}$ уточнением $G_{j}\left(G_{j}\right.$ укрупнением $\left.G_{i}\right)$, тогда и только тогда, когда для любого $x \in G_{i}$ существует $y \in G_{j}$, такое, что $x \subseteq y$; пусть $G_{i} \leq G_{j}$ означает, что $G_{i}$ является уточнением $G_{j}$.

$G_{i}$ называется непосредственным уточнением $G_{j}$ тогда и только тогда, когда не существует $G_{k} \in G_{n}$, такого, что $G_{i} \leq G_{k}$ и $G_{k} \leq G_{j}$. Для заданной структуры $G_{i} \in G_{n}$ структурное соседство определяется как множество всех непосредственных уточнений и непосредственных укрупнений $G_{i}$ в $G_{n}$. Рассмотренные структуры образуют решетку уточнения $G$-структур. Решетка может быть получена в результате неоднократного выполнения 
процедуры, порождающей все непосредственные уточнения для любой структуры из решетки.

Таким образом, структурированная система $\boldsymbol{S} F$ является реконструктивной гипотезой полной системы $\mathcal{G}$, если каждый ее элемент является подсистемой полной системы и выполняются два условия:

1) Условие покрытия:

$$
\bigcup_{k \in N_{q}}{ }^{k} V=V .
$$

2) Условие неизбыточности:

$$
\left(\forall j, k \in N_{q}\right)\left({ }^{j} V \subseteq{ }^{k} V \Longrightarrow j=k\right)
$$

Условие 1 продиктовано требованием использования всей информации о каждой переменной полной системы в реконструктивной гипотезе. Условие 2 позволяет отбросить из реконструктивной гипотезы избыточные элементы структурированной системы.

Близость двух сопоставимых систем с поведением рассматривается как потеря информации при замене $f_{\mathcal{G}}$ на $f_{S F}^{h}$. Количество информации относительно полной системы $\mathcal{G}$, потерянной в реконструктивной гипотезе $\boldsymbol{S F}$ может быть измерено при помощи информационного расстояния.

Для вероятностных систем информационное расстояние выражается известной формулой:

$$
D\left(f_{\mathcal{G}}, f_{S F}^{h}\right)=\frac{1}{\log _{2}|C|} \sum_{c \in C} f_{\mathcal{G}}(c) \log _{2} \frac{f_{\mathcal{G}}(c)}{f_{S F}^{h}(c)^{\prime}}
$$

где $f_{\mathcal{g}}(c), f_{s F}^{h}(c)$ - значение вероятности для состояния $c \in C$ в полной системе и в реконструкции полной системы $\boldsymbol{S} F$, соответственно; 
$\frac{1}{\log _{2}|c|}=$ const - нормирующий коэффициент, благодаря которому информационное расстояние обладает свойством: $0 \leq D\left(f_{\mathcal{G}}, f_{s}\right) \leq 1$.

Однако предложенные Дж. Клиром единые методы, основанные на обработке эмпирических данных, на практике имеют свои ограничения увеличение числа параметров системы приводит к высокой вычислительной сложности. Из-за критически быстрого роста количества реконструктивных гипотез, связанного с увеличением числа параметров, определяющих состояние системы, основатель системологии Дж. Клир не смог распространить разработанную им методику на сложные системы с большим количеством параметров. Однако наличие и силу взаимосвязей в системологии позволяют определить только методы структурированных систем при исследовании реконструктивных свойств. Для уменьшения вычислительной сложности и возможности применения методов структурирования систем при решении системных задач (в процессе принятия оптимальных решений) необходима разработка модифицированных процедур поиска решеток уточнения, отбора реконструктивных гипотез и др.

Для разрешения указанных трудностей, в рамках исследований Т.И. Синельниковой, выполняемых в Кубанском государственном университете под руководством к.ф.-м. наук, доц. Н.А. Швецовой, автором впервые предложена модификация алгоритма решения системных задач с применением структурированных систем [10, 11, 12]. В алгоритмы Дж. Клира было введено понятие целевой переменной, которое указывает на то, что поиск реконструктивных гипотез будет производиться в окрестности данной переменной (в реконструктивные гипотезы эти переменные обязательно должны входить, определяется влияние переменных системы на целевые переменные). Выбор целевой переменной (их может быть несколько) предоставляется пользователю программного 
модуля, целевыми не могут быть все переменные, входящие в систему. Проблема крайне быстрого роста количества реконструктивных гипотез с увеличением числа переменных в модифицированном алгоритме решается путем отсечения значительного количества реконструктивных гипотез, появляющихся на последующих этапах уточнения структурированных систем, которое основано на наложении ограничений на информационное расстояние между альтернативами и полной системой на каждом уровне уточнения. Модифицированный алгоритм решает проблемы, возникающие при использовании алгоритма Дж. Клира, что позволило осуществить его программную реализацию (свидетельство о государственной регистрации программы ЭВМ №2015618132).

Для работы модуля пользователь задает матрицу данных, автоматически производится переход от наборов состояний или частот встречаемости к вероятностной мере нечеткости, следствием которого является сформированная система с поведением (рисунок 1).

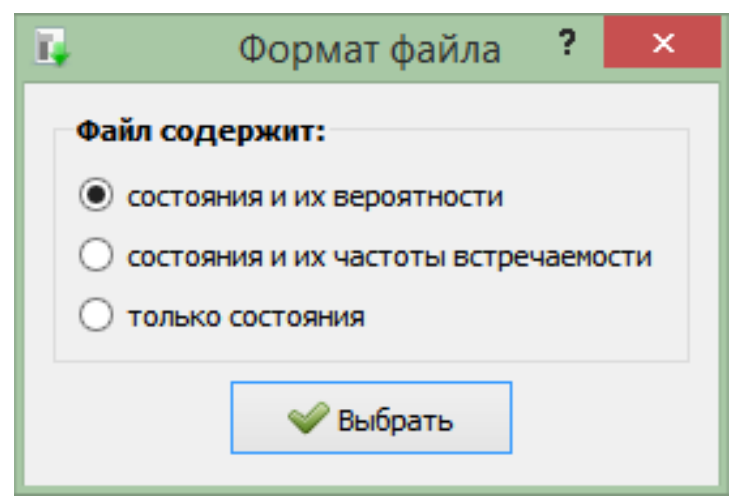

Рисунок 1. Выбор формата файла при загрузке.

Затем производится анализ реконструктивных свойств, включающий формирование структурированных систем, решение задачи идентификации, решение задачи реконструкции. Модуль включает математические процедуры сравнения вариантов решения (реконструктивных гипотез) и исключения из рассмотрения тех вариантов, которые не удовлетворяют заданным пользователем условиям. В результате структурирования систем и реконструктивного анализа 
получаем возможность произвести оптимальную декомпозицию системы, определить глубинные связи между элементами системы, а значит состояния переменных для желаемого развития системы. Полученные результаты доступны для отображения в графическом виде (рисунок 2, рисунок 3).

\begin{tabular}{|c|c|c|c|c|}
\hline \multirow[t]{2}{*}{ ए } & \multicolumn{2}{|l|}{ Расстояния } & \multirow[t]{2}{*}{$-\square$} & \multirow[t]{2}{*}{$x$} \\
\hline & Наборы & Расстояния & & \\
\hline Степень уточнения 2 & $123 / 124 / 134 / 234$ & $0.000010^{\star \star}$ & & \\
\hline Степень уточнения 3 & 123 / 124 / 134 & 0.000080 & & \\
\hline Степень уточнения 3 & 123 / 124 / 234 & $0.000020^{\star *}$ & & \\
\hline Степень уточнения 3 & 123 / 134 / 234 & 0.000120 & & \\
\hline Степень уточнения 3 & 124 / 134 / 234 & 0.000300 & & \\
\hline Степень уточнения 4 & $123 / 124 / 34$ & $0.000090^{\star \star}$ & & \\
\hline Степень уточнения 4 & $123 / 234 / 14$ & 0.000450 & & \\
\hline Степень уточнения 4 & $124 / 234 / 13$ & 0.000310 & & \\
\hline Степень уточнения 5 & $123 / 124$ & 0.010680 & & \\
\hline Степень уточнения 5 & $123 / 14 / 24 / 34$ & $0.000250^{* \star}$ & & \\
\hline Coxp & ить в файл & Закрыть & & \\
\hline
\end{tabular}

Рисунок 2. Информационные расстояния реконструктивных гипотез

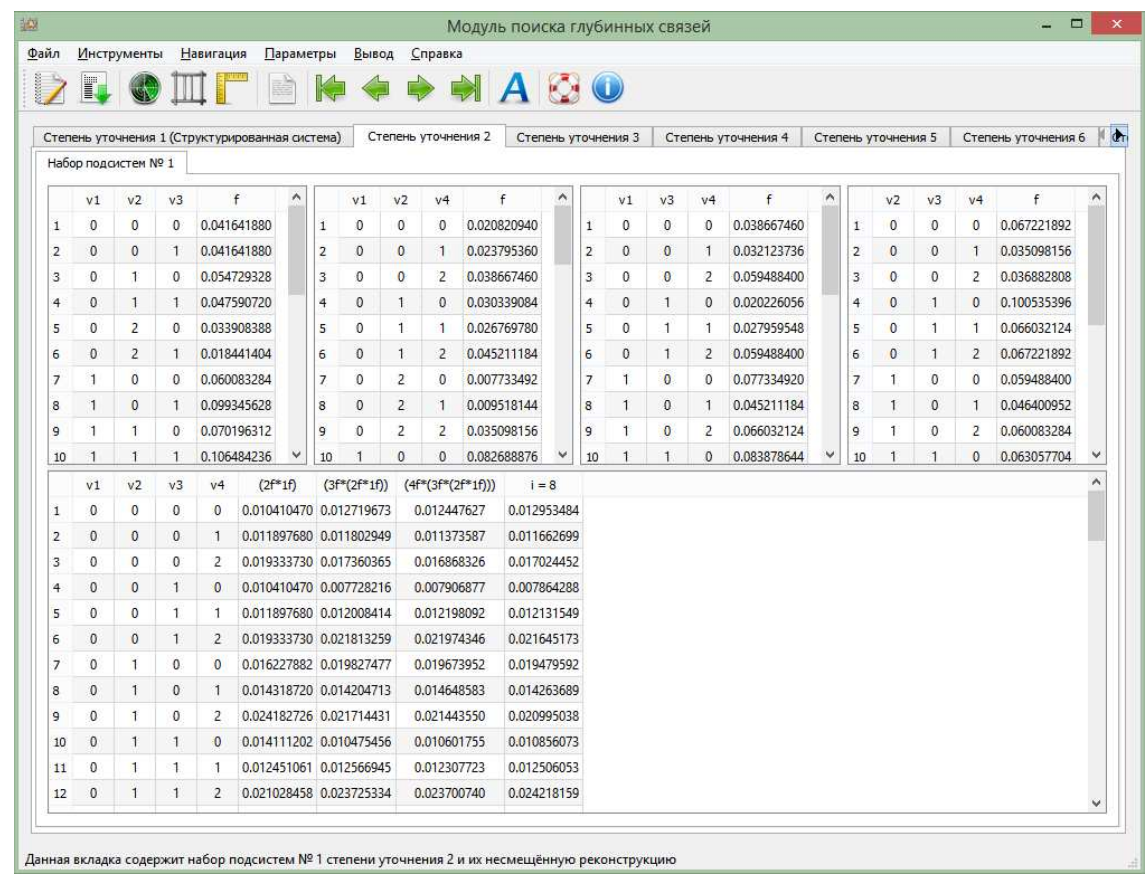

Рисунок 3. Результат выполнения реконструктивного анализа.

Программа работает под управлением операционных систем Windows ХР / 7 / 8.1/10. Разработанная система является открытой системой, возможно ее включение в другие более сложные системы, расширение 
дополнительными программными модулями, адаптация под платформы класса Unix, Mac OS.

На сегодняшней день в рамках исследований, проводимых Т.И. Синельниковой в Кубанском государственном университете под руководством к.ф.-м. наук, доц. Н.А. Швецовой, разработаны вычислительные алгоритмы методов исходных и порождающих систем системологии. Полученные результаты позволили разработать программный комплекс, реализующий указанные методы (свидетельство о государственной регистрации программы для ЭВМ: Российская федерация, номер свидетельства: №2016611326). Ведутся дальнейшие работы по их апробации и применению.

\section{Литература}

1. Богданов А.А. Тектология: (Всеобщая организационная наука). В 2-х кн.: Кн. 1. - М.: Экономика 1989. - 304 с.

2. Богданов А.А. Тектология: (Всеобщая организационная наука). В 2-х кн.: Кн. 2. - М.: Экономика 1989. - 351 с.

3. Берталанфи Л. Общая теория систем: критический обзор // Исследование по общей теории систем: сборник переводов. - М.: Прогресс, 1969. - С. 23-82.

4. Рапопорт А. Математические аспекты абстрактного анализа систем // Исследования по общей теории систем. Сборник переводов с польского и английского. - М.: Прогресс, 1969. - С. 83-105.

5. Росс-Эшби У. Введение в кибернетику / Пер. с англ. Д.Г. Лахути под ред. В.А. Успен-ского с предисловием А.Н. Колмогорова. - М.: Издательство иностранной литературы, 1959. - 432 с.

6. Boulding K. E. General Systems Theory - the Skeleton of a Science // Management Science, 1956, Vol. 2, No. 3, pp.197-208.

7. Винер Н. Кибернетика, или Управление и связь в животном и машине. / Пер. с англ. И.В. Соловьева и Г.Н. Поварова; Под ред. Г.Н. Поварова. - 2-е издание. - М.: Наука; Главная редакция изданий для зарубежных стран, 1983. - 344 с. - Электронная биб-лиотека «Библиотека Михаила Грачева» - (Рус.). - URL: http://grachev62.narod.ru/cybern/contents.htm [15 августа 2012].
8. George Klir.
[Электронный
pecypc] https://www.binghamton.edu/ssie/people/klir.html

9. Клир Дж. Системология. Автоматизация решения системных задач. - М.: Радио и связь, 1990. - 535с.

10. Швецова Н.А., Синельникова Т.И. Инструментальное средство для создания структурированных систем // Современное состояние и приоритеты развития фундаментальных наук в регионах: Труды VIII Научной конференции молодых учёных и студентов. - Краснодар: Просвещение-Юг, 2011. С. 136-138. 
11. Синельникова Т.И. О целесообразности использования компьютерных структурированных систем в процессе выработки оптимальных управленческих решений // Экономика знаний: проблемы управления формированием и развитием: материалы VI Междунар. науч.-практ. конф. - Краснодар: Кубанский гос. ун-т, 2014. С. 317-325.

12. Швецова Н.А., Синельникова Т.И. Методы системологии в системе поддержки принятия решений // Международный журнал экспериментального образования. - 2015. - № 11 - С. 136-137.

\section{References}

1. Bogdanov A.A. Tektologija: (Vseobshhaja organizacionnaja nauka). V 2-h kn.: Kn. 1. - M.: Jekonomika 1989. - $304 \mathrm{~s}$.

2. Bogdanov A.A. Tektologija: (Vseobshhaja organizacionnaja nauka). V 2-h kn.: Kn. 2. - M.: Jekonomika 1989. - $351 \mathrm{~s}$.

3. Bertalanfi L. Obshhaja teorija sistem: kriticheskij obzor // Issledovanie po obshhej teorii sistem: sbornik perevodov. - M.: Progress, 1969. - S. 23-82.

4. Rapoport A. Matematicheskie aspekty abstraktnogo analiza sistem // Issledovanija po obshhej teorii sistem. Sbornik perevodov s pol'skogo i anglijskogo. - M.: Progress, 1969. - S. 83-105.

5. Ross-Jeshbi U. Vvedenie v kibernetiku / Per. s angl. D.G. Lahuti pod red. V.A. Uspen-skogo s predisloviem A.N. Kolmogorova. - M.: Izdatel'stvo inostrannoj literatury, 1959. $-432 \mathrm{~s}$.

6. Boulding K. E. General Systems Theory - the Skeleton of a Science // Management Science, 1956, Vol. 2, No. 3, pp.197-208.

7. Viner N. Kibernetika, ili Upravlenie i svjaz' v zhivotnom i mashine. / Per. s angl. I.V. Solov'eva i G.N. Povarova; Pod red. G.N. Povarova. - 2-e izdanie. - M.: Nauka; Glavnaja redakcija izdanij dlja zarubezhnyh stran, 1983. - 344 s. - Jelektronnaja bib-lioteka «Biblioteka Mihaila Gracheva» - $\quad$ (Rus.). - URL: http://grachev62.narod.ru/cybern/contents.htm [15 avgusta 2012].
8. George
Klir.
[Jelektronnyj
resurs]

https://www.binghamton.edu/ssie/people/klir.html

9. Klir Dzh. Sistemologija. Avtomatizacija reshenija sistemnyh zadach. - M.: Radio i svjaz', 1990. - 535s.

10. Shvetsova N.A., Sinelnikova T.I. Instrumental'noe sredstvo dlja sozdanija strukturirovannyh sistem // Sovremennoe sostojanie i prioritety razvitija fundamental'nyh nauk v regionah: Trudy VIII Nauchnoj konferencii molodyh uchjonyh i studentov. Krasnodar: Prosveshhenie-Jug, 2011. S. 136-138.

11. Sinelnikova T.I. O celesoobraznosti ispol'zovanija komp'juternyh strukturirovannyh sistem v processe vyrabotki optimal'nyh upravlencheskih reshenij // Jekonomika znanij: problemy upravlenija formirovaniem i razvitiem: materialy VI Mezhdunar. nauch.-prakt. konf. - Krasnodar: Kubanskij gos. un-t, 2014. S. 317-325.

12. Shvetsova N.A., Sinelnikova T.I. Metody sistemologii v sisteme podderzhki prinjatija reshenij // Mezhdunarodnyj zhurnal jeksperimental'nogo obrazovanija. - 2015. - № $11-$ S. 136-137. 